\title{
Functional Voice Disorders and Personality
}

\section{Mafalda Andrea1, Maria Luísa Figueira²}

1 - Psychiatry Department, Santa Maria Hospital, Lisbon, Portugal; mafaldaandrea@gmail.com

2 - University Clinic of Psychiatric and Medical Psychology, Faculty of Medicine, University of Lisbon, Portugal

\section{Introduction and aims}

Functional voice disorders (FVD) are common vocal pathologies in the ENT outpatient clinics (10 to 40\%) and have a female predominance (3:1). These patients present apparently normal laryngeal anatomy and physiology, a reduced vocal fold amplitude (Figure 1) with significant impairment in vocal quality ${ }^{1,2}$.

Besides of their clinical relevance in the field of voice disorders, FVD are also of interest for mental health specialists due to the significant co-morbidity with anxiety, depression, personality disorders, somatization, and conversion reaction ${ }^{3}$.

The aim of this study was to analyse the relationship between personality and FVD.

\section{Materials and methods}

After the diagnosis of a FVD made by ENT specialists or residents through videolaryngoscopy, 83 female patients (mean age of 52.51 years) were screened with The Millon Multiaxial Inventory (MCMI-II) to identify the personality traits and disorders associated with FVD. The internal consistency was generally good with alpha values ranging from 0.62 (histrionic) to 0.89 (borderline).

\section{Results}

The most common personality disorders presented in our sample were obsessive-compulsive (63.9\%), dependent (37.3\%) and avoidant $(19.3 \%)$, while the most frequent personality traits were schizoid (26.5\%), narcissistic (25.3\%), histrionic $(24.1 \%)$ and avoidant (20.5\%) (Figure 2).

\section{Conclusions}

Our findings revealed an association between FVD and personality. The high incidence of personality disorders identified in this study highlights the importance of its assessment.

Personality evaluation has clinical implications in the etiopathogenesis of FVD as well as in the prevention of treatment compliance of these patients. We suggest that the vocal multidisciplinary assessment should integrate not only ENT specialists and speech therapists but also mental health specialists.

\section{REFERENCES}

1. Andrea, M., Dias, O., Andrea, M., \& Figueira, M.L. Functional Voice Disorders: The Importance of the Psychologist in Clinical Voice Assessment. Journal of Voice. 2017; 31 (4): 507.e13-507.e22.

2. Baker, J. (2017). Psychosocial perspectives on the management of voice disorders. Implications for clients options and strategies for clinicians. Oxford: Compton Publishing.

3. Rosen D, Sataloff R. Psychological aspects of voice disorders. In: Sataloff R, ed. Vocal Health and Pedagogy. San Diego, CA: Singular Publishing Group; 1997:243-255. 